\title{
Introducing Variability into Aspect-Oriented Modeling Approaches
}

\author{
Philippe Lahire ${ }^{2}$, Brice Morin ${ }^{1}$, Gilles Vanwormhoudt ${ }^{3}$, Alban Gaignard ${ }^{2}$, \\ Olivier Barais ${ }^{1}$, and Jean-Marc Jézéquel ${ }^{1}$
}

${ }^{1}$ IRISA Rennes, Projet Triskell, IRISA - Campus de Beaulieu F-35042 Rennes Cedex

${ }^{2}$ I3S Nice-Sophia Antipolis, Equipe Rainbow, I3S-UNSA

Les algorithmes, 2000 route des lucioles BP 121 F-06903 Sophia-Antipolis Cedex

${ }^{3}$ GET Telecom-Lille 1/ LIFL, Université de Lille 1, F-59655 Villeneuve d'Ascq Cedex

\begin{abstract}
Aspect-Oriented Modeling (AOM) approaches propose to model reusable aspects, or cross-cutting concerns, that can be composed in different systems at a model or code level. Building complex systems with reusable aspects helps managing software complexity. But in general, reusability of an aspect is limited to a particular context. On the one hand, if the target model does not match the template point-to-point, the aspect cannot be applied. On the other hand, even when it is actually applied, it is woven into the target model always in the same way. In this paper 1 , we point out the needs of variability in the AOM approaches and introduce seamless variability mechanisms in an existing AOM approach to improve reusability. Our aspects can fit various contexts and can be composed into the base model in different ways. Introducing variability into AOM approaches will turn standard aspects into highly reusable aspects.
\end{abstract}

\section{Introduction}

The Aspect Oriented Software Development (AOSD) paradigm first appeared at the code level a decade ago [7] with the most famous AOP language AspectJ 6]. The aspect paradigm offers a new way to construct complex systems by composing crosscutting concerns with the base system. In the earlier stages of the software life-cycle, several Aspect-Oriented Modeling approaches (AOM) already exist [12416], with various levels of abstraction (requirement, design, architecture). In general, these approaches decrease the complexity of systems by composing models that represents the different concerns of the system (business, security, persistence ...). To help developers saving time designing systems and therefore reduce the time-to-market of these systems, models should be reusable.

Currently, AOM approaches provide some means to design reusable and flexible aspects. But, reusability and flexibility are often limited. In general, they

\footnotetext{
${ }^{1}$ This work was partially supported by the French National Research Agency
}

(RNTL FAROS Project). 
describe one possible variant of an aspect and propose one possible way to integrate it. For example, a designer cannot model a design pattern in its full genericity with these approaches: he can only model one specific implementation choice for this design pattern. Consequently, aspects are only reusable in similar or very related contexts. In this paper, we argue that aspects must be reusable in various contexts. Designing context independent aspects requires seamless variability mechanisms for specifying the weaving, the pointcut expression, etc... . Such mechanisms will turn standard aspects into highly reusable and flexible pieces of models. The contribution of this paper is to point out the needs of variability in the AOM approaches, to provide some mechanisms to support variability in one particular AOM approach and to illustrate these new mechanisms on a concrete example. To address variability in software development, Software product lines (SPL) offer some mechanisms to support functional variability 2 and to derive products that match the user's needs. However, this variability only concerns the software module specifications. In the case of AOM approaches, variability should also be applied onto the composition mechanisms.

The remainder of this paper is organized as follows. Section 2 points out the needs of variability in the AOM approaches with a motivating example. Section 3 presents an overview of an AOM approach. This approach is extended in the section 4 to support variability mechanisms. Section 5 describes a metamodel for this approach and the implementation of a modeling tool. Section 6 presents related works and section 7 concludes and discusses future work.

\section{Motivating Example}

To illustrate the needs of variability in the AOM approaches, we use the example of a mobile phone device. Figure 1 shows a simplified class diagram presenting the main functionalities of an accountancy package for a mobile phone.

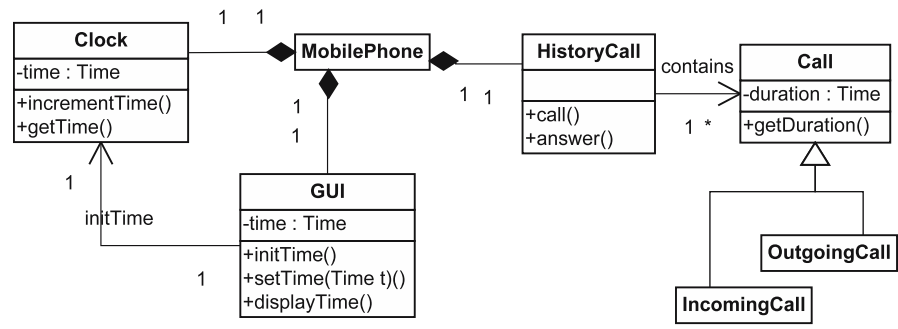

Fig. 1. A Simplified class diagram of the mobile phone

When the user is calling (resp. is called by) someone, the HistoryCall class creates a new outgoing (resp. incoming) call and saves the duration. The class GUI can display its local variable time which is initialized when switching on the phone. The class Clock only contains a variable time which is incremented every minute.

\footnotetext{
${ }^{2}$ see Software Product Line Conferences : http://www.splc.net
} 


\subsection{Matching Variability}

Two optional requirements, total calls and total outgoing calls, can be added to our mobile phone in order to compute the total duration of the (outgoing) calls. We will use the Counter pattern [1] to realize these two requirements.

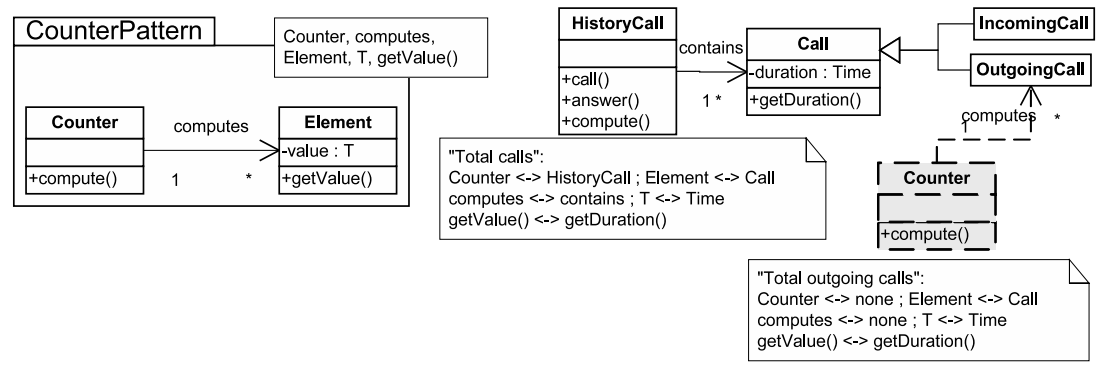

Fig. 2. The Counter pattern realizing the total calls requirement

In most of the AOM approaches [21116], a template specifies the model elements of the concern that have to be bound with target model elements. Reusability is then limited to iso-structural target models because if the structure does not match the template point-to-point, the aspect cannot be applied.

Figure 2 shows the Counter pattern composition into the mobile phone model. In order to realize the total calls requirement, we use the existing HistoryCall and Call classes to respectively act as Counter and Element. We now want to realize the total outgoing calls requirement in a separate Counter class. This requires the creation of a new class acting as a Counter and the insertion of a new association between this class and the OutgoingCall class. Instead of modifying the base model to this end, it would be more efficient that the Counter pattern automatically introduces all these missing elements. However this is not possible with classic AOM approaches [21116] because the weaving process of the aspect upon the base system can not vary depending on the bindings.

\subsection{Adaptation Variability}

One optional requirement, display time, can be added in order to display and update the time every minute, when the internal clock is updated. The Observer pattern will realize this requirement, notifying the GUI (Observer) that the Clock (Subject) has been updated.

In most of the AOM approaches 21116, aspects are composed into the target model using one composition rule at a time, offering poor flexibility. Depending on the context, it would be very useful to easily switch between different composition rules. In the context of embedded systems it may be preferable to reduce the number of classes because of memory limitations, and completely merge the aspect while in some other cases, it may be preferable to compose the aspect 

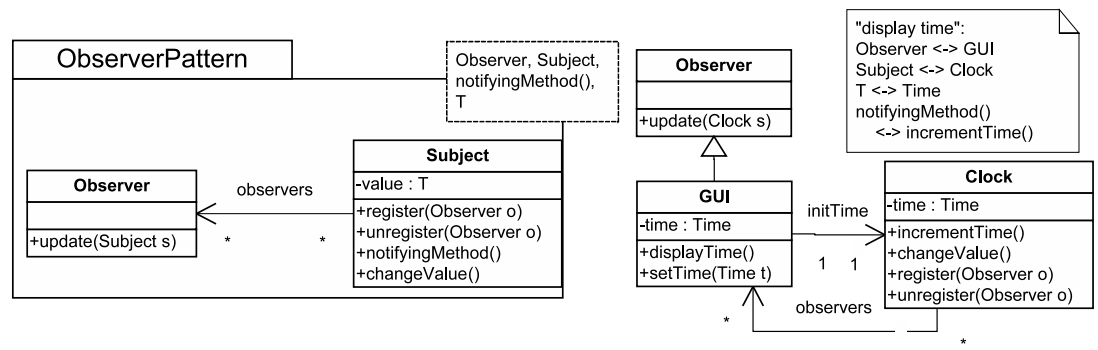

Fig. 3. The Observer pattern merged into the base model

by inheritance in order to improve readability. Figure 3 illustrates another composition rule where Subject is merged into Clock whereas GUI inherits from Observer.

This motivating example has shown the needs of variability in two contexts i.e. matching and adaptation. There is also a need for functional variability e.g. how to design many versions of the Counter pattern (total or average for example). Since Software Product Line approaches [17. can help modeling this kind of variability, we do not cover them into this paper.

\section{An AOM Approach Overview}

The approach which is presented in this paper is only one among many possible approaches for addressing AOM [311. It focuses on providing capabilities for concerns (functional or extra-functional) to be reused. In this context, the expressiveness of the concern modeling is not a primary objective. For example, contrary to other non aspect-oriented approaches like [17, we do not offer more capabilities for expressing the variability of concerns than the one provided by the underlying metamodel used for the concern specification. The approach called SmartAdAPTERs had been applied first to Java programs [8] and more recently to EMF models. It leverages the notions of subject [14] and aspect programming 677. Its key concepts are concerns, adapters, adaptations and adaptation target. The main idea is the following: each concern identified as reusable should go with an adapter which specifies a composition protocol, that is a set of adaptations and adaptation targets describing how the concern should be composed with other concerns when it is reused. This protocol will guide the designer to identify the specific parts for reuse when composing a reusable concern into a target concern.

We propose to explain this approach through the reuse of the Observer design pattern. First we define its composition protocol (see Figure 4). For better readability, we use a concrete textual syntax in order to specify this composition protocol. Details in the concrete syntax are not important and the syntax might be slightly modified in the future. 
01 concern designpattern.observer

02 abstract adapter ObserverAdapter \{

03

04 abstract Class target "class(es) representing an observer" : observerClass

05 abstract Class target "class(es) representing a subject" : subjectClass

06 abstract Method target " method(s) notifying changes" : notifyingMethod

07 require notifyingMethod in subjectClass.*

adaptation becomeObserver "Modify class to make it an observer" :

inherit Observer in observerClass

adaptation becomeSubject "Modify class in order to make it a subject" : merge class subjectClass with Subject

adaptation introduceLink "introduce an association (subject to observer) " : introduce Association observers (subjectClass - $i$ observerClass)

adaptation notifyingObserver

"Alter notifyingMethods to tell observers about modification" :

extend method notifyingMethod (...) with after $\{$ changeValue ()$;\}$

abstract adaptation updateObserver "add an update facility to observers" :

introduce method public void update(subjectClass s) in Observer

... Protocol includes also :object initialization,observers registration,...

$26\}$

Fig. 4. Snippet of the composition protocol for the Observer design pattern

Let us now detail this example illustrated in Figure 4 Line 01 specifies the concern to be reused. The adapter called ObserverAdapter describes its composition protocol (Line 02). When the composition protocol is defined the concern(s) that may reuse it are not known so that we do not know the classes corresponding to the objects acting as subjects and those acting as observers. The only thing that we may assume is that there are classes that act as observers and subjects. They are represented by the two abstract targets of type class: observerClass and subjectClass (Lines 04 and 05). Each of these targets may be associated to one or several classes at composition time.

Considering the design pattern Observer of Figure 3, any subject must inform an observer that its content has been modified by calling the method changeValue. For the same reasons that the classes mentioned above are not known the method(s) playing this role are also not known but they should exist and be declared in the subjectClass (Lines 06 and 07 ). To ensure that the call to changeValue is performed by the method(s) notifyingMethod, the composition protocol specifies an adaptation of type interception which adds this call at the end of the corresponding method(s) (Lines 18 to 20 ).

More generally this kind of adaptation deals with some actions to be taken when a classifier member (attributes, methods...) is accessed or called. These adaptations allow the designer to add behavior at the beginning, end or around some existing methods but also to add some treatment when an exception is triggered. For attributes, interception may occur when the attribute is read or modified.

Let us continue with our example. To be able to call changeValue or any other feature of class Subject, it is necessary to have access to it from within the classes corresponding to subjectClass. This means that we have to specify another adaptation. Two possibilities could be chosen: to merge all the features 
of class Subject into subjectClass, or to make subjectClass inherit from Subject. Here we choose an adaptation of type Merging (Lines 12 and 13).

Such adaptations deal mainly with packages, classifiers features and associations. Method merging is particularly interesting if there is a support for describing the behavior (programming constructs in KeRmeta [13, Sequence diagrams in UML, etc.). At present time merging policies are mainly execution of one method before the other; the handling of interlaced method bodies could be inspired by approaches like 9]. Merging classifiers is either straightforward (no conflict, name of features to be merged are identical, feature appear only in one of the classes,...) or may need more information in order to relate the features of the classifiers that need to be merged [2]16].

All these adaptations were dealing with the subjects. It is then necessary to address observers and to also insert class Observer at the right place(s) in the target concern. We chose here to inherit from it (Lines 09 and 10). Such adaptation is of type Introduction. It deals not only with superclass introduction as it is the case here but also with adding classifier members (new attributes or methods), as well as association. It is also possible to add a classifier invariant or a method assertion (Precondition or postcondition).

We use the same type of adaptation to insert the association between subject and observer classes as specified in the design pattern. Depending on the association to be introduced we may provide additional information. For example, in the current case the association is unidirectional from subjects to observers (Lines 15 and 16).

It only remains one thing to do: to add to the observerClass class(es) a method update (also an adaptation of type Introduction), that reacts to the changes made in the subject object. At this time we do not know the content of this feature because we do not know what the purpose of the target concern is. This is why the adaptation is abstract. The advantage to plan this adaptation in the composition protocol is to guide and control the reuse of the design pattern.

This composition protocol continues with the description of the initialization and the registration of observers but for space reasons we do not include it.

Let us suppose now that this concern is reused by the concern described in Figure 1 (Section 2) dealing with mobile phones. So we need to compose these two concerns. The information which is imcomplete into the composition protocol (abstract targets and adaptations) is described into a concrete adapter ApplicationPhone which specializes the adapter ObserverAdapter as it is shown in Figure 5] Please note that, in this example, the insertion is in situ. It means that adaptations are performed within the concern application.phone. In some cases, it is better to make the composition ex situ that is to say to compose the two concerns into a new one.

In the above composition protocol (Figure 4) we made several assumptions about the target concern. For example, we suppose that the association does not yet exist between the classes GUI (the observer) and Clock (the subject). This is a drawback because if the composition does not deal with a concern which satisfies these assumptions, it will be impossible to reuse the composition 


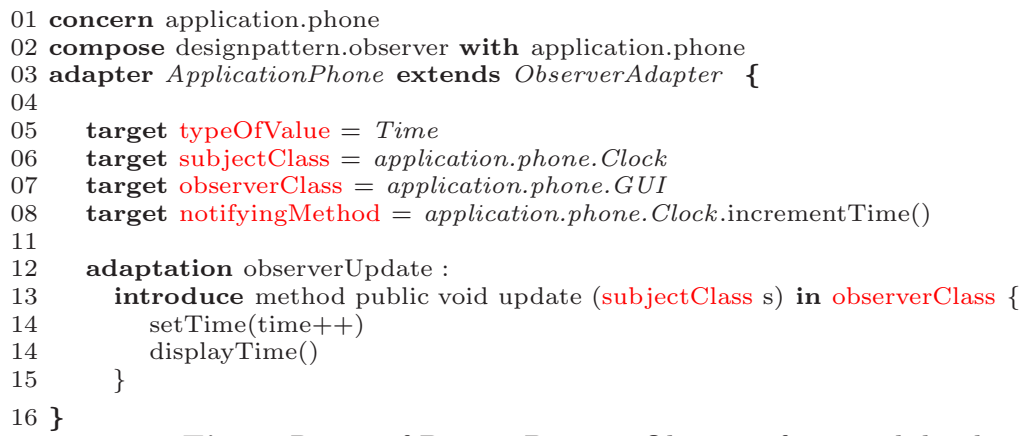

Fig. 5. Reuse of Design Pattern Observer for a mobile phone

protocol in another context. Thus, we reach the conclusion that we need to introduce some variability within the composition protocol. This is the purpose of section 4

\section{Extension to Support Variability}

In Section 3 we proposed an overview of the SMARTADAPTERS approach. We now consider the needs of variability pointed out in Section 2, Our objective in this section is to introduce matching and adaptation variability into the composition protocol in order to make it more reusable and as consequence to make the concern itself more reusable. SMARTADAPTERS is a support for explaining our approach but we plan to address other AOM approaches. Variability mechanisms introduced are inspired by Software Product Lines approaches, especially [17.

Figure 6] shows what we should introduce in an adapter to better customize the composition protocol. In Section 5 we will describe the metamodel containing the capabilities that are suggested here.

We may note first that adapter ObserverAdapter is now preceded by the keyword derivable (Line 02). This means that it may present several alternatives to implement the composition and may consider some adaptation targets or adaptations as optional. This adapter acts as a template where some information should be given in order to choose between possible variants or options.

A first possible customization is dealing with the insertion of the features provided by classes Subject and Observer. Depending on the target concern or more generally on the context of reuse, it may be interesting to have the choice between inheriting from those classes or merging their features into observerClass and subjectClass. In Figure 4 a choice is made a priori. In Figure 6, the choice is described by the Lines 09 to 24 through a clause Alternative Insertion Choices which specifies here two variants (more variants could be defined if needed). A variant may contain several adaptation target declarations and adaptations. Implicitly this means that these targets and adaptations are dependent from each others.

Now, we can introduce the update method. If we merge the Subject and the $O b$ server, we need to introduce the update method in the class where the Observer is merged i.e., observerClass (Lines 22 and 23). Subject is also merged in a target 


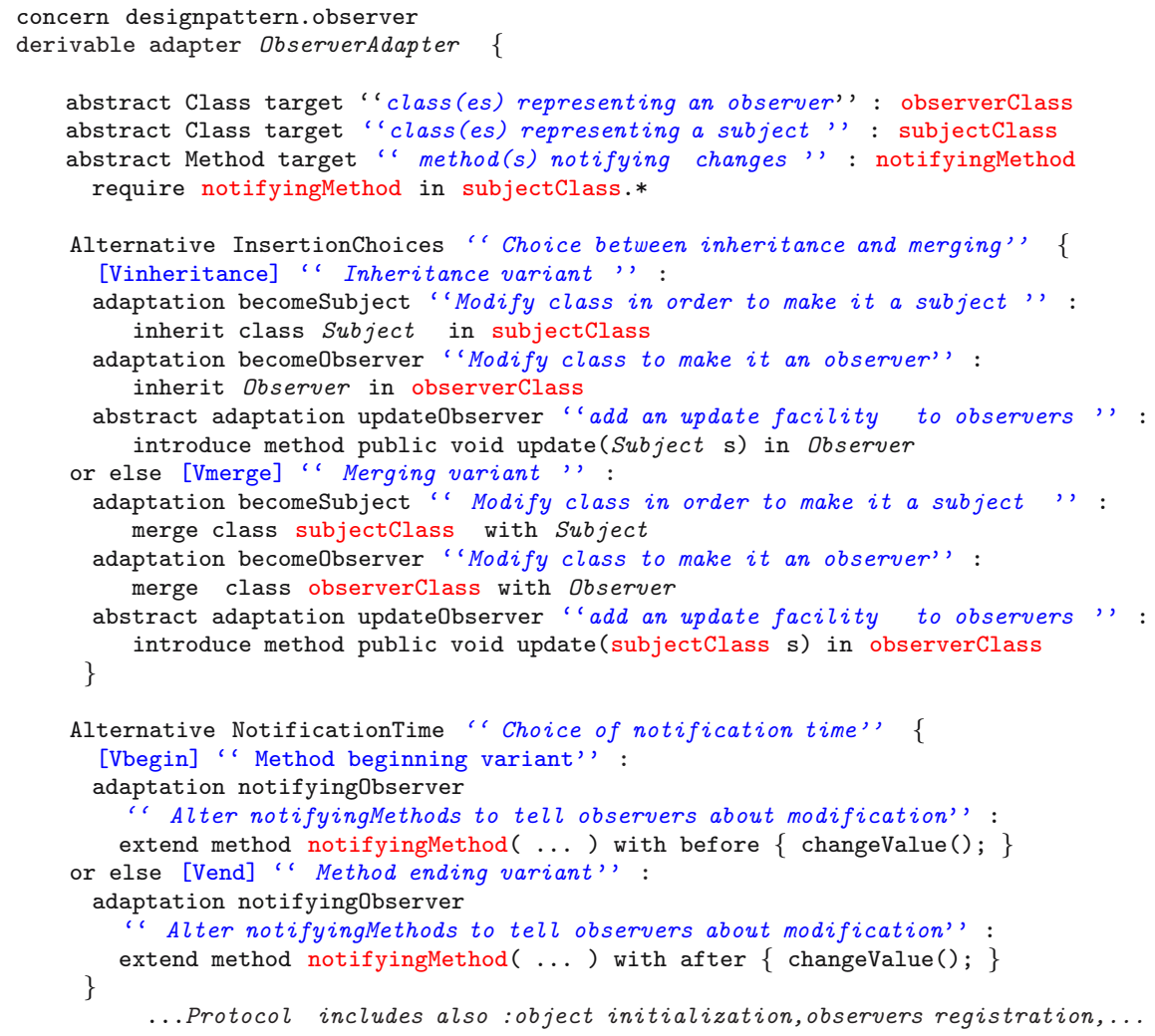

Fig. 6. Composition protocol for the Observer with variability

class, therefore the parameter of the update method has the type of this target class i.e. subjectClass. If the pattern is composed by inheritance, the update method is introduced in the Observer class itself, and the parameter has the type Subject (Lines 15 and 16). The update method is very related to the composition variant, so we integrate its introduction in the InsertionChoices alternative. Depending on the chosen composition variant, the right update method will be introduced. In both cases the contents of this method is not already known, that is why this method is abstract.

A second possible customization is related to the location of the call to method change Value within notifyingMethod. It may be useful depending on the target concern to notify the subject changes to observers either at the beginning or at the end of the execution of notifyingMethod. The corresponding variants are described by the Lines 22 to 31 through a second clause Alternative. Each variant corresponds to a unique adaptation of type Interception.

In figure 7 we extend this protocol to experiment the combination of optional and constraint clauses. We now address the association between observers and subjects (called observers in the design pattern of Figure2). It is very likely that 


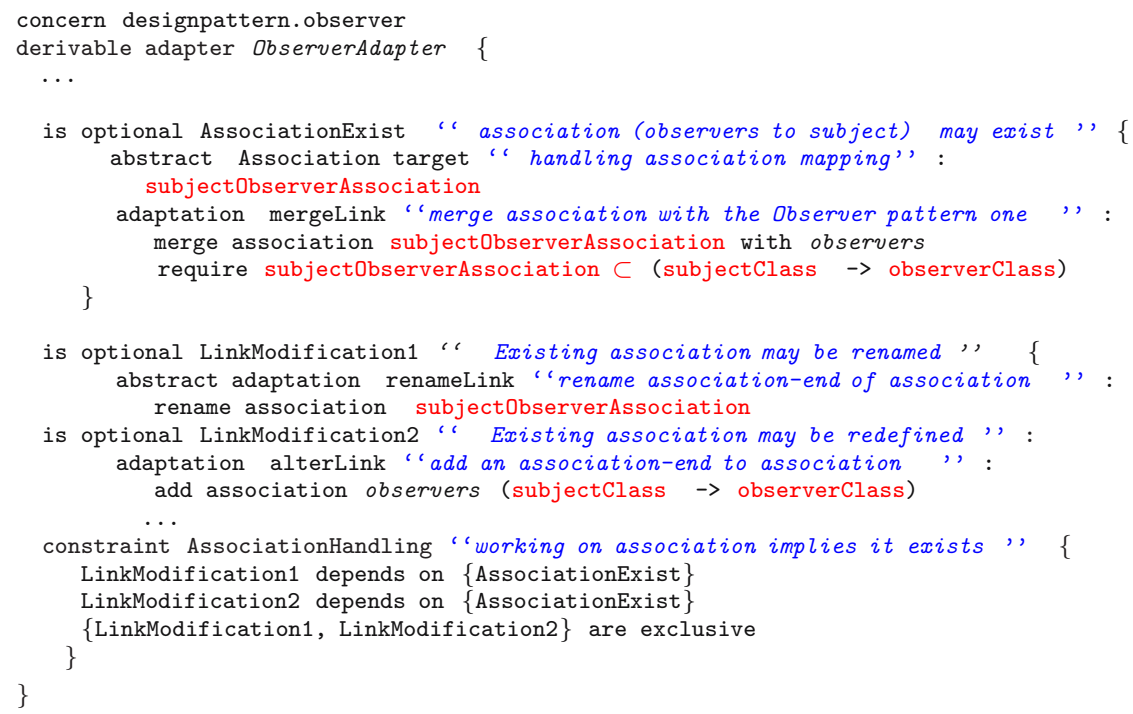

Fig. 7. Options and matching variability

depending on the target concern this association may already exist in it. In order to authorize both situations we propose some optional adaptations (Lines 05 to 18). A first optional clause assumes that the association exists in the target concern and is identified by the target subjectObserverAssociation; it must be merged with observers. Then it may be possible to specify a renaming adaptation because nothing can ensure that it has the same association-end name in the target concern. It is also possible to add an association-end when the association exists but in the opposite way in this concern.

The example developed in Figures [6] and 7 especially illustrates the needs for optional parts and variant definitions. In order to insure the consistency of the composition protocol, the user can define mutual exclusion and dependency constraints. These constraints restrict the number of possible combinations to sensible ones. In our example, we want to ensure that $i$ ) renaming and redefinition may not be performed if the association between observers and subjects does not exists in the target concern and, ii) renaming its association-end is incompatible with adding observers. These contraints are expressed (Figure 7- Lines 20 to 23) by introducing dependencies between LinkModification1, LinkModification2 and AssociationExists options and a mutual exclusion between the first two options.

Now, we can compose the variable "Design Pattern Observer" into the mobile phone base model. In addition to the tasks described in figure 5 it is necessary to select options and variants (adaptation targets and adaptations) which are suitable for the concern "mobile phone". Of course the abstract adaptation targets and adaptations to concretize in the adapter ApplicationPhone depends on the variants and options which are selected (Figure 8).

The selection is made through a clause derive (Lines 05 to 08 ). No association can match the observers association in the target model, so the optional clauses 


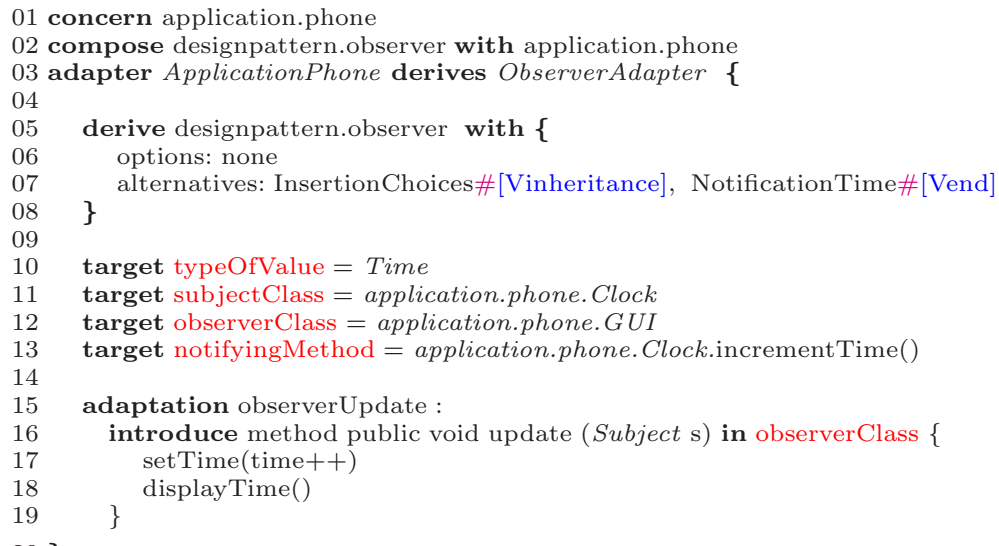

Fig. 8. Reuse of Design Pattern Observer for a mobile phone

are not selected (note that an association exists in application.phone but in the opposite way so that it would be possible to keep only one association selecting AssociationExist and LinkModification2). We also select the two variants associated to the alternative clauses Insertion Choices and NotificationTime. Finally, we have to concretize the update method, specifying that the GUI has to increment its variable time and refresh the screen. Concretizing abstract methods in a concrete adapter is close to the mechanism defined in the AOP approach of Hannemann et al. 5]. Mandatory targets and adaptations of Figure 6 are processed normally in the same way as it is done in Figure 5.

Figure 9 shows two types of composition i.e, merging and inheritance, in order to realize the display time requirement. Inheritance corresponds to the adapter we have derived above, while Merging corresponds to another possible derivation provided by the protocol.
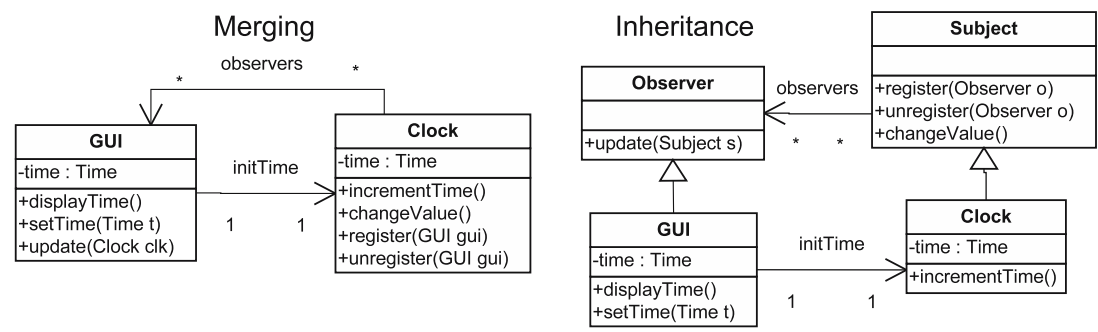

Fig. 9. Two possible compositions of the Observer pattern

In the motivating example, we were not able to realize the total outgoing calls with the standard Counter pattern because the template approach was not flexible enough. We can now realize the total calls and the total outgoing calls requirements using the same Counter pattern. Indeed, the Counter pattern now can be applied either if the class acting as Counter is present or not in the base model. For 
space limitation, the derivable adapter and the concrete adapter are not shown but the principle is similar to the Observer protocol (Figures 6, 7]and 8).

Finally, it is interesting to note that introducing variability did not affect the guidance and the controls when reusing a derivable concern. On the contrary, the choices induced by the addition of variability is also controlled and guided thanks to the expressiveness of the composition protocol.

\section{Metamodeling and Implementing AOM with Variability}

This section proposes a metamodel of concerns that includes concepts for adapters and variability illustrated in sections 3 and 4 . This metamodel aims at giving a precise formulation of concerns and make it possible their integration into modeling tools. Figure 10 shows an excerpt of the metamodel where concepts introduced to handle variability are identified with a circle at the upper left. The key concepts of the metamodel are concern, adapter, target and adaptation.

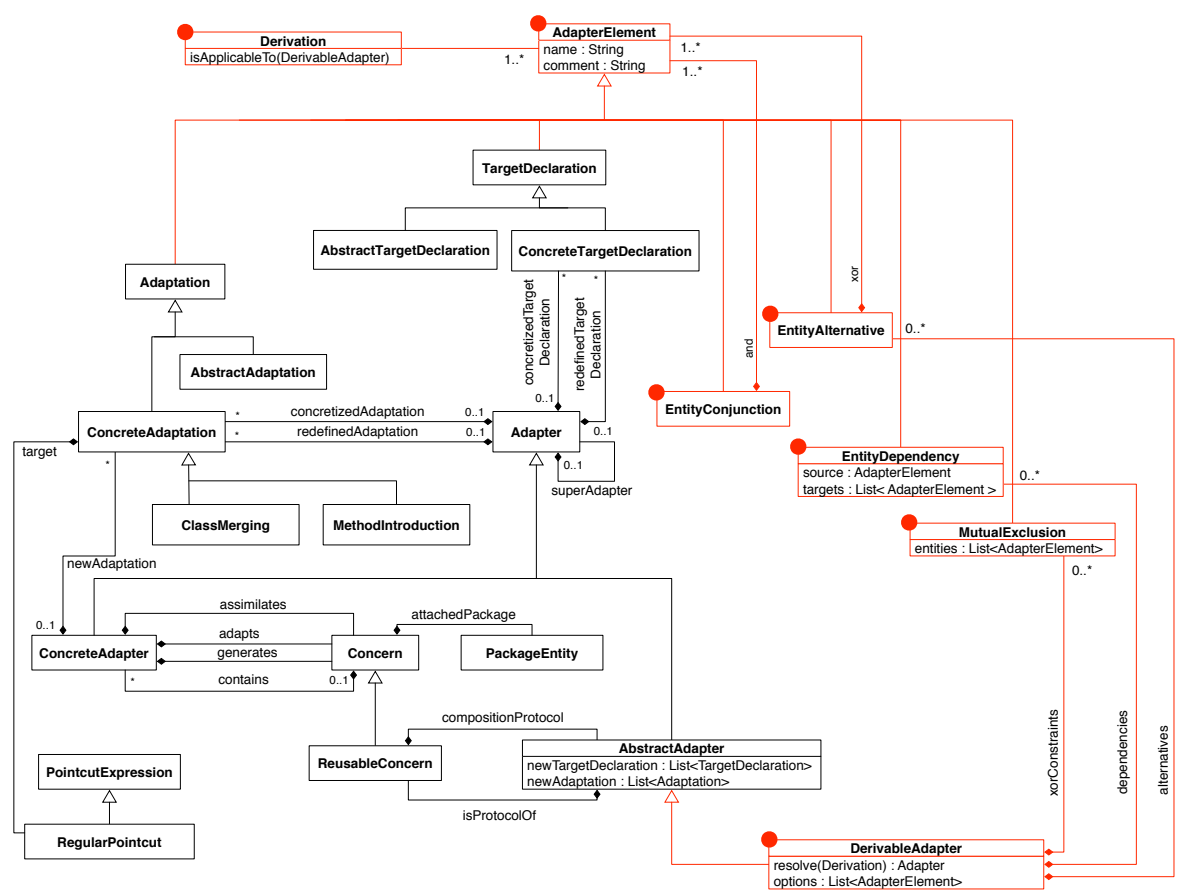

Fig. 10. Metamodel of SmartAdapter with variability

A reusable concern (class ReusableConcern) is associated to a package (class PackageEntity) which contains the concern description 3 and its protocol of composition (class AbstractAdapter). Concerns are not always reusable (class Concern).

\footnotetext{
${ }^{3}$ We assume that a concern is described by a package of classes similarly to a UML class diagram.
} 
For example the concern which describes the GUI of an application is rather specific and may not be reusable; such concerns do not have a composition protocol but could be composed with other concerns. A concern refers to as many concrete adapters (ConcreteAdapter) as there are concern to be integrated with it.

An adapter (class Adapter) is identified by a name and may inherit (i.e. specialize) from another adapter. An adapter may be abstract (class AbstractAdapter), concrete (class ConcreteAdapter) or derivable (class DerivableAdapter). Each adapter contains adaptations (class Adaptation) and adaptation targets (class TargetDeclaration). A derivable adapter is an abstract adapter which supports variability: it allows the designer to customize the set of adaptations or/and the set of adaptation targets by expressing options, alternatives, dependencies and exclusions. Such an adapter is not intended to be used directly for composing concerns but serves to derive an adapter. A derived adapter is obtained using the method resolve of class DerivableAdapter which takes a derivation (class Derivation) parameter to select the adaptations and the adaptation targets among the options and variants. This adapter may be concrete, abstract or derivable depending on what is resolved by the derivation parameter.

A target declaration (class TargetDeclaration) specifies an adaptation target that matches the entities on which the adaptations relies on. An adaptation target may identify just one required element (class AbstractTargetDeclaration) (like the observers or the subjects in the design pattern Observer) or be fully specified (class ConcreteTargetDeclaration) by referencing the real element (class, method, ...) to adapt.

An adaptation (class Adaptation) specifies the action to be taken for an element of the reusable concern when it is composed. The metamodel includes a hierarchy of adaptation classes that are typed according to the types of target entities (package, classifier, method, attributes and association) and reflect the four kinds of adaptation currently proposed: interceptions, introduction, merging and redefinitions. Figure 10 shows two of the adaptation classes used in the previous examples (class ClassMerging and class MethodIntroduction).

To be able to take into account several variants for the integration of the concern, the metamodel includes the concept of alternative entity (EntityAlternative). An alternative entity may refer to several adaptations or adaptation targets (see xor link) but only one will be selected at composition time.

Adaptations, adaptation targets and even alternatives can be optional in a derivable adapter, that is to say that they are planned in the composition protocol but they could be retained or not when the concern is composed with another one. Optional elements of a derivable adapter are referenced by its options link.

Practically several adaptations or adaptations targets may be described in a given variant or be declared as an optional block. For this purpose we propose a way to group thoses entities (class EntityConjunction).

In a derivable adapter, classes EntityDependency and MutualExclusion allows designers to specify that an AdapterElement (variant or option) may not be selected with other ones or on the contrary must be selected if some others are selected. These classes define constraints that are checked before deriving a derivable 
adapter, in order to insure the consistency of the derived adapter. If a derivation does not respect these constraints then an exception is raised that asks the user to modify the derivation.

The metamodel described above has been used to build a modeling tool integrated in the Eclipse environment. This tool currently provides two main functionalities: designing models of concerns and adapters; composing concerns from their models. This tool has been implemented using the Eclipse Modeling Framework (EMF) and the Kermeta language [13. We have exploited EMF to define a Ecore version of our metamodel, reusing the Ecore metamodel for the description of concerns. The Kermeta language has been exploited to extend the Ecore version of our metamodel with operational behavior. This behavior performs several tasks related to the design and composition of concerns: it checks the consistency of adapters, computes derived adapters and compose elements of concerns from a set of adaptations. At this time, we are investigating the design of a concrete textual syntax for our metamodel like the one used in the previous section and we plan to build the concrete syntax tool using a meta-model centric approach as [12].

\section{Related Work}

There exists numerous AOM approaches but few of them support variability mechanisms at the composition level 4161]. In 2, Clarke et al. model an aspect in a template package specifying the structure and the behavior of the aspect with a class diagram and sequence diagrams. The template is composed of model elements present in the concern's class diagram and specifies the elements that have to be matched in the target model. There is no functional or matching variability mechanism. The composition relationship authorizes multiple bindings i.e. it is possible to match several target model elements to the same concern model element. Adaptation lacks variability: concerns are indeed always merged into the target model. Note that it is possible to generate AspectJ code to postpone the weaving at code level. Our adaptation protocol allows the designer to define different variants of how the concern will be integrated in the target model. All the variability mechanisms we have identified may be adapted to Theme.

Muller et al. [11] also propose an approach to compose a reusable model expressed as a template package with an existing model. To express this composition, they introduce an apply operator that specifies the mapping between template parameters and elements of the target model. Their approach addresses variability at the composition level by giving the capacity to annotate the apply operator with different strategies such as "merge" or "view". Strategies are only provided to get different resulting models. Compared to our proposal, this solution does not offer any mechanism to express options and variants for the reusable model. It is also less flexible as it does not offer finer grain mechanisms to control how elements of reusable and target models must be composed.

France et al. [16] have developed a systematic approach for composing class diagrams in which a default composition procedure based on name matching can be customized by user-defined composition directives. These directives constrain 
how class diagrams are composed. The framework automatically identifies conflicts between models that have to be composed and it solves them thanks to the composition directives. Contrary to Theme, composition directives address the weaving only from the structural point of view. They consider the composition as a model transformation. The variability can be addressed by designing several composition directives depending on the integration context. However, the definition of the composition directive would then become messy and error-prone. Besides, it is a symmetric AOM approach in which they do not differentiate between aspect model and base model. Consequently, they do not currently provide a pointcut language to manage the composition.

In [5], Hannemann et al. propose an AOP approach to implement design patterns with AspectJ. They propose up to seven different implementations for each design pattern. The only variability mechanism is the generalization relationship between an abstract aspect and an aspect. For example, the update method of the Observer is declared abstract in an abstract aspect and its contents will be specified in a concrete aspect. We also use this mechanism but the variability mechanisms we introduced allow a concern to be applied in multiple contexts whereas we would have to create a new aspect depending on the context with the Hannemann et al. approach. Option and variant notions do not exist, reducing the reusability of the aspects. Our concerns are adaptable and do not need modifications to be applied, but only customization. Introducing the same variability mechanisms at the code level code could enhance the expressiveness of AOP language such as AspectJ.

\section{Conclusion}

In this work, we propose an approach for introducing variability in aspect-oriented modeling (AOM). To achieve this goal, two important parts of such an AOM approach were needed: A concern model and a weaver that support variability. In this paper we mainly focus on the second one. Indeed, the variability in the concern specification depends on the expressiveness of the meta-model dedicated to concern modeling. Consequently, a reasonable solution to integrate variability in the concern model can be inspired by product lines researches and more precisely by 17 .

To introduce variability in the weaving process, the composition meta-model of our AOM approach has been extended. These extensions concern the adaptations primitives and the pointcut specification. They are composed of a set of entities specifying optional parts, alternatives, dependencies and mutual exclusion constraints. These extensions allow the user to design a family of aspects at the design level that can be derived to be applied in a particular context.

One of the main benefits of building a composition protocol is the capability to control and guide the software architect when he designs new applications. The variability introduction does not affect the guidance and the control when reusing a derivable concern. On the contrary, the choices induced by the addition of variability are also controlled and guided thanks to the expressiveness of the composition protocol. 
In the SMARTADAPTERS platform, we plan to improve the pointcut language and the target identification. One possible solution is to describe the pointcut with a template model and to use pattern matching [15] to identify targets. We also want to generalize the SMARTADAPTERS to various metamodels, not only class diagrams or Java programs. In [10], we have proposed and implemented a metamodel-driven approach to generate domain-specific AOM frameworks that uses the aforementioned pointcut language. Finally, AOM approaches can be used to manage variability in software product line. Our work can be merged to these approaches to show why variability is also needed in the aspects in order to use an AO approach to build software product line.

\section{References}

1. Aldawud, O., Elrad, T., Bader, A.: UML Profile for Aspect-Oriented Software Development. In: 3rd International Workshop on Aspect Oriented Modeling (In conjunction of AOSD'03), Boston, Massachusetts (March 2003)

2. Baniassad, E., Clarke, S.: Theme: An Approach for Aspect-Oriented Analysis and Design. In: ICSE '04. Proceedings of the 26th International Conference on Software Engineering, pp. 158-167. IEEE Computer Society, Washington, DC, USA (2004)

3. Barais, O., Le Meur, A.F., Duchien, L., Lawall, J.: Safe integration of new concerns in a software architecture. In: ECBS '06. Proceedings of the 13th Annual IEEE International Symposium and Workshop on Engineering of Computer Based Systems, pp. 52-64. IEEE Computer Society, Washington, DC, USA (2006)

4. Elrad, T., Aldawud, O., Bader, A.: Aspect-Oriented Modeling: Bridging the Gap between Implementation and Design. In: Batory, D., Consel, C., Taha, W. (eds.) GPCE 2002. LNCS, vol. 2487, pp. 189-201. Springer, Heidelberg (2002)

5. Hannemann, J., Kiczales, G.: Design Pattern Implementation in Java and Aspectj. In: OOPSLA '02. Proceedings of the 17th ACM SIGPLAN conference on Objectoriented programming, systems, languages, and applications, pp. 161-173. ACM Press, New York, NY, USA (2002)

6. Kiczales, G., Hilsdale, E., Hugunin, J., Kersten, M., Palm, J., Griswold, W.G.: An Overview of Aspectj. In: Knudsen, J.L. (ed.) ECOOP 2001. LNCS, vol. 2072, pp. 327-353. Springer, Heidelberg (2001)

7. Kiczales, G., Lamping, J., Menhdhekar, A., Maeda, C., Lopes, C., Loingtier, J-M., Irwin, J.: Aspect-Oriented Programming. In: Aksit, M., Matsuoka, S. (eds.) ECOOP 1997. LNCS, vol. 1241, pp. 220-242. Springer, Heidelberg (1997)

8. Lahire, Ph., Quintian, L.: New Perspective To Improve Reusability in ObjectOriented Languages. Journal Of Object Technology (JOT) 5(1), 117-138 (2006)

9. Mens, T., Kniesel, G., Runge, O.: Transformation Dependancy Analysis, a Comparison of two Approaches. In: Rousseau, R., Urtado, C., Vauttier, S. (eds.) Proceedings of LMO 2006, Langages et Modèles à Objets, Nîmes, France, pp. 167-182. HermesLavoisier (Mars 2006)

10. Morin, B., Barais, O., Jézéquel, J.M., Ramos, R.: Towards a Generic Aspect-Oriented Modeling Framework. In: 3rd International Workshop on Models and Aspects (In conjunction of ECOOP'07), Berlin, Germany (2007)

11. Muller, A., Caron, O., Carré, B., Vanwormhoudt, G.: On Some Properties of Parameterized Model Applications. In: Proceedings of ECMDA'05: First European Conference on Model Driven Architecture - Foundations and Applications, Nuremberg, Germany (November 2005) 
12. Muller, P.A, Fleurey, F., Fondement, F., Hassenforder, M., Schneckenburger, R., Gérard, S., Jézéquel, J.M: Model-driven analysis and synthesis of concrete syntax. In: Nierstrasz, O., Whittle, J., Harel, D., Reggio, G. (eds.) MoDELS 2006. LNCS, vol. 4199, pp. 98-110. Springer, Heidelberg (2006)

13. Muller, P.A., Fleurey, F., Jezequel, J.M.: Weaving Executability into ObjectOriented Meta-languages. In: Briand, L.C., Williams, C. (eds.) MoDELS 2005. LNCS, vol. 3713, Springer, Heidelberg (2005)

14. Ossher, H., Tarr, P.: Hyper/J: Multi-Dimentionnal Separation of Concern for Java. In: Ghezzy, C. (ed.) Proceedings of ICSE'00, Limerick, Ireland, ACM Press, New York (2000)

15. Ramos, R., Barais, O., Jézéquel, J.M.: Matching model-snippets. In: MoDELS '07. Model Driven Engineering Languages and Systems, 10th International Conference, Nashville, Tennessee (2007)

16. Reddy, Y.R., Ghosh, S., France, R.B., Straw, G., Bieman, J.M., McEachen, N., Song, E., Georg, G.: Directives for Composing Aspect-Oriented Design Class Models. In: Rashid, A., Aksit, M. (eds.) Transactions on Aspect-Oriented Software Development I. LNCS, vol. 3880, pp. 75-105. Springer, Heidelberg (2006)

17. Ziadi, T., Jézéquel, J.M.: Families Research Book. In: Product Line Engineering with the UML: Products Derivation. LNCS, pp. 557-588. Springer, Heidelberg (2006) 